\title{
Existence of nontrivial solutions for asymptotically linear periodic Schrödinger equations
}

\author{
Shaowei Chen * Dawei Zhang \\ School of Mathematical Sciences, Huaqiao University, \\ Quanzhou 362021, P. R. China
}

\begin{abstract}
We study the Schrödinger equation:

$$
-\Delta u+V(x) u=f(x, u), \quad u \in H^{1}\left(\mathbb{R}^{N}\right),
$$

where $V$ is periodic and $f$ is periodic in the $x$-variables, 0 is in a gap of the spectrum of the operator $-\Delta+V$ and $f$ is asymptotically linear as $|u| \rightarrow+\infty$. We prove that under some asymptotically linear assumptions for $f$, this equation has a nontrivial solution. Our assumptions for $f$ are different from the classical assumptions raised by $\mathrm{Li}$ and Szulkin.
\end{abstract}

Key words: Semilinear Schrödinger equations; linking; asymptotically linear.

2000 Mathematics Subject Classification: 35J20, 35J60

\section{Introduction and statement of results}

In this paper, we consider the following Schrödinger equation:

$$
-\Delta u+V(x) u=f(x, u), \quad u \in H^{1}\left(\mathbb{R}^{N}\right),
$$

where $N \geq 1, V(x)$ is continuous and periodic in $x_{j}$ for $j=1, \cdots, N, 0$ is in a gap of the spectrum of the operator $-\Delta+V$ and $f \in C\left(\mathbb{R}^{N} \times \mathbb{R}\right)$ is periodic in $x_{j}$ and asymptotically linear as $|u| \rightarrow \infty$.

Semilinear Schrödinger equations with periodic coefficients have attracted considerable attention over the past decade. Because of its natural variational structure (see (2.4) in Section 2 of this paper), critical point theory is the main method obtaining solutions to Eq. (1.1). When $V$ is bounded below by a positive constant, the operator $-\Delta+V$ is positive definite. In this case, classical theorems in critical point theory, such as the mountain pass theorem (see, for example, [30]), can be used to obtain solutions to Eq.(1.1) (see the classical paper [4, 11] and the more recent paper [16]). However, when 0 is in a gap of the spectrum of the operator $-\Delta+V$, this operator has an infinitely dimensional negative space, and the classical linking theorems (e.g., [30]) can not be applied. To overcome this difficulty, some new infinite-dimensional linking theorems were developed (see [6, 13, 23, 27]). Using these generalized linking theorems, many results on the existence and multiplicity of nontrivial solutions for (1.1) have been obtained (see [17, 14, 22, 26, 32, 33]). In [13], Kryszewski and Szulkin proved that (1.1) has a nontrivial solution if $f$ satisfies the Ambrosetti-Rabinowitz condition, and has infinitely many solutions if

${ }^{*}$ E-mail address: swchen6@163.com (Shaowei Chen) 
the additional assumption that $f$ is odd holds. In [15], Li and Szulkin obtained a nontrivial solution for (1.1) if $f$ satisfies some asymptotically linear assumptions, and in [6], Ding proved that if $f$ is odd, then, under the same assumptions as in [15], (1.1) has infinitely many geometrically different solutions. In [23] (see also [22]), Schechter and Zou combined a generalized linking theorem with the monotonicity methods of Jeanjean (see [11]). They obtained a nontrivial solution of (1.1) when $f$ exhibts the critical growth. A similar approach was applied by Szulkin and Zou to obtain homoclinic orbits of asymptotically linear Hamiltonian systems (see [27]). Finally, we should point out that, although these generalized linking theorems have achieved great success in strongly indefinite problems, there are other approaches that can be used to deal with (1.1) effectively. For instance, see [1, 3, 5, 9, 18, 19, 29] and the references therein.

In [15], Li and Szulkin studied Eq.(1.1) under the following assumptions:

(v). $V \in C\left(\mathbb{R}^{N}\right)$ is 1 -periodic in $x_{j}$ for $j=1, \cdots, N$ and 0 is in a spectral gap $\left(\mu_{-1}, \mu_{1}\right)$ of $-\Delta+V$. Denote

$$
\mu_{0}:=\min \left\{-\mu_{-1}, \mu_{1}\right\}
$$

$\left(\mathbf{f}_{\mathbf{1}}\right) . f \in C\left(\mathbb{R}^{N} \times \mathbb{R}\right)$ is 1 -periodic in $x_{j}$ for $j=1, \cdots, N$ and $f(x, t) / t \rightarrow 0$ as $t \rightarrow 0$ uniformly in $x \in \mathbb{R}^{N}$.

$\left(\mathbf{f}_{2}\right) \cdot f(x, t)=V_{\infty}(x) t+f_{\infty}(x, t)$, where $V_{\infty}$ and $f_{\infty}$ are 1-periodic in $x_{j}$ for $j=1, \cdots, N$,

$$
f_{\infty}(x, t) / t \rightarrow 0 \quad \text { uniformly in } \quad x \in \mathbb{R}^{N} \text { as }|t| \rightarrow \infty,
$$

and $V_{\infty}(x) \geq \mu$ for all $x$ and some $\mu>\mu_{1}$.

$\left(\mathbf{f}_{3}\right) . \widetilde{F}(x, t):=\frac{1}{2} t f(x, t)-F(x, t) \geq 0$ for all $(x, t) \in \mathbb{R}^{N} \times \mathbb{R}$, where $F(x, t)=\int_{0}^{t} f(x, s) d s$.

$\left(\mathbf{f}_{\mathbf{4}}\right)$. There exists $\delta \in\left(0, \mu_{0}\right)$ such that if $f(x, t) / t \geq \mu_{0}-\delta$, then $\widetilde{F}(x, t) \geq \delta$.

Under assumption $\left(\mathbf{f}_{\mathbf{1}}\right)$, the zero function $u=0$ is obviously a trivial solution of (1.1). Therefore we focus on finding nontrivial solutions, namely solutions $u$ of (1.1) such that $u \neq \equiv 0$ in $\mathbb{R}^{N}$. In [15], Li and Szulkin obtained a nontrivial solution of equation (1.1) under the above assumptions by applying the generalized linking theorem (see [13] or [30, Chapter 6]). After [15], conditions similar to $\left(\mathbf{f}_{\mathbf{4}}\right)$ have become classical assumptions for strongly indefinite problems with asymptotically linear nonlinearities (see, for example, [6] and [7]).

We consider Eq.(1.1) under assumptions different to $\left(\mathbf{f}_{\mathbf{4}}\right)$. More precisely, we assume:

$\left(\mathbf{v}^{\prime}\right) .0$ is not in the spectrum of the operator

$$
\begin{aligned}
& T_{2}: L^{2}\left(\mathbb{R}^{N}\right) \rightarrow L^{2}\left(\mathbb{R}^{N}\right), \quad u \mapsto-\Delta u+\left(V-V_{\infty}\right) u, \\
& \text { with domain } D\left(T_{2}\right):=\left\{u \in L^{2}\left(\mathbb{R}^{N}\right) \mid T_{2} u \in L^{2}\left(\mathbb{R}^{N}\right)\right\} .
\end{aligned}
$$

$\left(\mathbf{f}_{\mathbf{4}}^{\prime}\right)$. There exist $\kappa>0$ and $v \in\left(0, \mu_{0}\right)$ such that, for every $(x, t) \in \mathbb{R}^{N} \times \mathbb{R}$ with $|t|<\kappa$,

$$
|f(x, t)| \leq v|t|
$$

and for every $(x, t) \in \mathbb{R}^{N} \times \mathbb{R}$ with $|t| \geq \kappa$,

$$
\widetilde{F}(x, t)>0 .
$$

$\left(\mathbf{f}_{\mathbf{5}}^{\prime}\right) . \widetilde{F}(x, t)>0$ for all $(x, t) \in \mathbb{R}^{N} \times(\mathbb{R} \backslash\{0\})$.

Our main results are as follows: 
Theorem 1.1. Suppose $(\mathbf{v}),\left(\mathbf{v}^{\prime}\right),\left(\mathbf{f}_{\mathbf{1}}\right)-\left(\mathbf{f}_{\mathbf{3}}\right)$, and $\left(\mathbf{f}_{\mathbf{4}}^{\prime}\right)$ are satisfied. Then Eq.(1.1) has a nontrivial solution.

It is easy to verify that $\left(\mathbf{f}_{\mathbf{5}}^{\prime}\right)$ and the assumption that $f(x, t) / t \rightarrow 0$ as $t \rightarrow 0$ uniformly in $x \in \mathbb{R}^{N}$ imply $\left(\mathbf{f}_{\mathbf{4}}^{\prime}\right)$. Therefore, we have the following corollary:

Corollary 1.2. Suppose $(\mathbf{v}),\left(\mathbf{v}^{\prime}\right),\left(\mathbf{f}_{\mathbf{1}}\right),\left(\mathbf{f}_{\mathbf{2}}\right)$, and $\left(\mathbf{f}_{\mathbf{5}}^{\prime}\right)$ are satisfied. Then Eq.(1.1) has a nontrivial solution.

Remark 1.3. There are many functions satisfying $\left(\mathbf{f}_{\mathbf{4}}^{\prime}\right)$ or $\left(\mathbf{f}_{\mathbf{5}}^{\prime}\right)$ that do not satisfy $\left(\mathbf{f}_{\mathbf{4}}\right)$. An example of such a function $f$ can be constructed as follows: Let $b \in \mathbb{R}$ be such that $\frac{2}{3} b \notin \sigma\left(T_{2}\right)$ and $\frac{2}{3} b>\mu_{1}$, where $\sigma\left(T_{2}\right)$ denotes the spectrum of the operator $T_{2}$ defined by (1.2). Let

$$
F(x, t)=\frac{b t^{2}}{3}\left(1-\frac{1}{(1+|t|)^{3}}\right) \text { and } f(x, t)=F_{t}^{\prime}(x, t)=\frac{2 b}{3} t\left(1-\frac{1}{(1+|t|)^{3}}\right)+\frac{b t^{2} s g n t}{(1+|t|)^{4}} .
$$

It is easy to verify that

$$
\widetilde{F}(x, t)=\frac{b|t|^{3}}{2(1+|t|)^{4}}>0
$$

for all $(x, t) \in \mathbb{R}^{N} \times(\mathbb{R} \backslash\{0\})$. However, as $|t| \rightarrow+\infty, f(x, t) / t \rightarrow \frac{2}{3} b>\mu_{0}$ and $\widetilde{F}(x, t) \rightarrow 0$. Therefore, $f$ satisfies $\left(\mathbf{f}_{\mathbf{5}}^{\prime}\right)$, but does not satisfy $\left(\mathbf{f}_{\mathbf{4}}\right)$.

We use the generalized linking theorem for a class of parameter-dependent functionals (see [23, Theorem 2.1] or Proposition 2.2 in this paper) to obtain a sequence of approximate solutions for (1.1). Then, applying the main theorem in [10], we prove that these approximate solutions are bounded in $L^{\infty}\left(\mathbb{R}^{N}\right)$ and $H^{1}\left(\mathbb{R}^{N}\right)$ (see Lemma 3.1 and 3.3). These are the two most important steps in our proof. Finally, using the concentration-compactness principle, we obtain a nontrivial solution of (1.1).

Notation. $B_{r}(a)$ denotes the open ball of radius $r$ and center $a$. For a Banach space $E$, we denote the dual space of $E$ by $E^{\prime}$, and denote strong and weak convergence in $E$ by $\rightarrow$ and $\rightarrow$, respectively. For $\varphi \in C^{1}(E ; \mathbb{R})$, we denote the Fréchet derivative of $\varphi$ at $u$ by $\varphi^{\prime}(u)$. The Gateaux derivative of $\varphi$ is denoted by $\left\langle\varphi^{\prime}(u), v\right\rangle, \forall u, v \in E . L^{p}\left(\mathbb{R}^{N}\right)$ denotes the standard $L^{p}$ space $(1 \leq p \leq \infty)$, and $H^{1}\left(\mathbb{R}^{N}\right)$ denotes the standard Sobolev space with norm $\|u\|_{H^{1}}=$ $\left(\int_{\mathbb{R}^{N}}\left(|\nabla u|^{2}+u^{2}\right) d x\right)^{1 / 2}$. We use $O(h), o(h)$ to mean $|O(h)| \leq C|h|, o(h) /|h| \rightarrow 0$ as $|h| \rightarrow 0$.

\section{Existence of approximate solutions for Eq.(1.1)}

Under the assumptions $(\mathbf{v}),\left(\mathbf{f}_{\mathbf{1}}\right)$, and $\left(\mathbf{f}_{\mathbf{2}}\right)$, the functional

$$
\Phi(u)=\frac{1}{2} \int_{\mathbb{R}^{N}}|\nabla u|^{2} \mathrm{~d} x+\frac{1}{2} \int_{\mathbb{R}^{N}} V(x) u^{2} \mathrm{~d} x-\int_{\mathbb{R}^{N}} F(x, u) \mathrm{d} x
$$

is of class $C^{1}$ on $X:=H^{1}\left(\mathbb{R}^{N}\right)$, and the critical points of $\Phi$ are weak solutions of (1.1).

Assume that $(\mathbf{v})$ holds, and let $S=-\Delta+V$ be the self-adjoint operator acting on $L^{2}\left(\mathbb{R}^{N}\right)$ with domain $D(S)=H^{2}\left(\mathbb{R}^{N}\right)$. By virtue of $(\mathbf{v})$, we have the orthogonal decomposition

$$
L^{2}=L^{2}\left(\mathbb{R}^{N}\right)=L^{+}+L^{-}
$$

such that $S$ is negative (resp.positive) in $L^{-}$(resp.in $L^{+}$). As in [6, Section 2] (see also [7, Chapter 6.2]), let $X=D\left(|S|^{1 / 2}\right)$ be equipped with the inner product

$$
(u, v)=\left(|S|^{1 / 2} u,|S|^{1 / 2} v\right)_{L^{2}}
$$


and norm $\|u\|=\left|\left\|\left.S\right|^{1 / 2} u\right\|_{L^{2}}\right.$, where $(\cdot, \cdot)_{L^{2}}$ denotes the inner product of $L^{2}$. From $(\mathbf{v})$,

$$
X=H^{1}\left(\mathbb{R}^{N}\right)
$$

with equivalent norms. Therefore, $X$ continuously embeds in $L^{q}\left(\mathbb{R}^{N}\right)$ for all $2 \leq q \leq 2 N /(N-2)$ if $N \geq 3$ and for all $q \geq 2$ if $N=1,2$. In addition, we have the decomposition

$$
X=X^{+}+X^{-}
$$

where $X^{ \pm}=X \cap L^{ \pm}$is orthogonal with respect to both $(\cdot, \cdot)_{L^{2}}$ and $(\cdot, \cdot)$. Therefore, for every $u \in X$, there is a unique decomposition

$$
u=u^{+}+u^{-}, u^{ \pm} \in X^{ \pm}
$$

with $\left(u^{+}, u^{-}\right)=0$ and

$$
\int_{\mathbb{R}^{N}}|\nabla u|^{2} d x+\int_{\mathbb{R}^{N}} V(x) u^{2} d x=\left\|u^{+}\right\|^{2}-\left\|u^{-}\right\|^{2}, u \in X .
$$

Moreover,

$$
\mu_{-1}\left\|u^{-}\right\|_{L^{2}}^{2} \leq\left\|u^{-}\right\|^{2}, \quad \forall u \in X
$$

and

$$
\mu_{1}\left\|u^{+}\right\|_{L^{2}}^{2} \leq\left\|u^{+}\right\|^{2}, \quad \forall u \in X .
$$

The functional $\Phi$ defined by (2.1) can be rewritten as

$$
\Phi(u)=\frac{1}{2}\left(\left\|u^{+}\right\|^{2}-\left\|u^{-}\right\|^{2}\right)-\Psi(u),
$$

where $\Psi(u)=\int_{\mathbb{R}^{N}} F(x, u) \mathrm{d} x$.

Let $\left\{e_{k}^{ \pm}\right\}$be the total orthonormal sequence in $X^{ \pm}$. Let $P: X \rightarrow X^{-}, Q: X \rightarrow X^{+}$be the orthogonal projections. We define

$$
\|\mid u\|=\max \left\{\|Q u\|, \sum_{j=1}^{\infty} \frac{1}{2^{k+1}}\left|\left(P u, e_{k}^{-}\right)\right|\right\}
$$

on $X$. The topology generated by $\||\cdot|\|$ is denoted by $\tau$, and all topological notation related to it will include this symbol.

Definition 2.1. Let $\psi \in C^{1}(X ; \mathbb{R})$. A sequence $\left\{u_{n}\right\} \subset X$ is called a Cerami sequence at level $c$ $\left((C)_{c}\right.$-sequence for short) for $\psi$, if $\psi\left(u_{n}\right) \rightarrow c$ and $\left(1+\left\|u_{n}\right\|\right)\left\|\psi^{\prime}\left(u_{n}\right)\right\| X_{X^{*}} \rightarrow 0$ as $n \rightarrow \infty$.

For $K>1$ and $\lambda \in[1, K]$, let

$$
\Phi_{\lambda}(u)=\frac{1}{2} \int_{\mathbb{R}^{N}}\left(|\nabla u|^{2}+V_{+}(x) u^{2}\right) d x-\lambda\left(\frac{1}{2} \int_{\mathbb{R}^{N}} V_{-}(x) u^{2} d x+\Psi(u)\right), u \in X,
$$

where $V_{ \pm}(x)=\max \{ \pm V(x), 0\}, \forall x \in \mathbb{R}^{N}$. It is easy to verify that a critical point $u$ of $\Phi_{\lambda}$ is a weak solution of

$$
-\Delta u+V_{\lambda}(x) u=\lambda f(x, u), u \in X
$$

where $V_{\lambda}=V^{+}-\lambda V^{-}$.

Let $R>r>0$ and $u_{0} \in X^{+}$with $\left\|u_{0}\right\|=1$. Set

$$
N=\left\{u \in X^{+} \mid\|u\|=r\right\}, M=\left\{u \in X^{-} \oplus \mathbb{R}^{+} u_{0} \mid\|u\| \leq R\right\} .
$$

Then, $M$ is a submanifold of $X^{-} \oplus \mathbb{R}^{+} u_{0}$ with boundary $\partial M$. 
Proposition 2.2 (Theorem 2.1 of [23]). Let $K>1$. The family of $C^{1}$-functional $\left\{H_{\lambda}\right\}$ has the form

$$
H_{\lambda}(u)=I(u)-\lambda J(u), u \in X, \lambda \in[1, K]
$$

Assume

(a) $J(u) \geq 0, \forall u \in X$,

(b) $|I(u)|+J(u) \rightarrow+\infty$ as $\|u\| \rightarrow+\infty$,

(c) for all $\lambda \in[1, K], H_{\lambda}$ is $\tau$-sequentially upper semi-continuous, i.e., if $\left\|\mid u_{n}-u\right\| \| \rightarrow 0$, then

$$
\limsup _{n \rightarrow \infty} H_{\lambda}\left(u_{n}\right) \leq H_{\lambda}(u)
$$

and $H_{\lambda}^{\prime}$ is weakly sequentially continuous. Moreover, $H_{\lambda}$ maps bounded sets to bounded sets,

(d) there exist $u_{0} \in X^{+} \backslash\{0\}$ with $\left\|u_{0}\right\|=1$, and $R>r>0$ such that for all $\lambda \in[1, K]$,

$$
\inf _{N} H_{\lambda}>\sup _{\partial M} H_{\lambda}
$$

Then there exists $E \subset[1, K]$ such that the Lebesgue measure of $[1, K] \backslash E$ is zero and for every $\lambda \in E$, there exist $c_{\lambda}$ and a bounded $(C)_{c_{\lambda}}$-sequence for $H_{\lambda}$, where $c_{\lambda}$ satisfies

$$
\sup _{M} H_{\lambda} \geq \sup _{\lambda \in E} c_{\lambda} \geq \inf _{\lambda \in E} c_{\lambda} \geq \inf _{N} H_{\lambda}
$$

Using this proposition and following the same argument as the proof of Corollary 3.4 of [27], we have the following lemma:

Lemma 2.3. Suppose that $(\mathbf{v})$ and $\left(\mathbf{f}_{\mathbf{1}}\right)-\left(\mathbf{f}_{\mathbf{3}}\right)$ hold. Then, there exist $K_{*}>1$ and $E \subset\left[1, K_{*}\right]$ such that the Lebesgue measure of $\left[1, K_{*}\right] \backslash E$ is zero and, for every $\lambda \in E$, there exist $c_{\lambda}$ and $a$ bounded $(C)_{c_{\lambda}}$-sequence for $\Phi_{\lambda}$, where $c_{\lambda}$ satisfies

$$
+\infty>\sup _{\lambda \in E} c_{\lambda} \geq \inf _{\lambda \in E} c_{\lambda}>0
$$

Proof. For $u \in X$, let

$$
I(u)=\frac{1}{2} \int_{\mathbb{R}^{N}}\left(|\nabla u|^{2}+V_{+}(x) u^{2}\right) d x
$$

and

$$
J(u)=\frac{1}{2} \int_{\mathbb{R}^{N}} V_{-}(x) u^{2} d x+\Psi(u) .
$$

Then, $I$ and $J$ satisfy assumptions $(a)$ and $(b)$ in Proposition 2.2, and, by (2.5), $\Phi_{\lambda}(u)=I(u)-$ $\lambda J(u)$.

From (2.5), for $u \in X$,

$$
\begin{aligned}
\Phi_{\lambda}(u) & =\frac{1}{2} \int_{\mathbb{R}^{N}}\left(|\nabla u|^{2}+V(x) u^{2}\right) d x-\frac{\lambda-1}{2} \int_{\mathbb{R}^{N}} V_{-}(x) u^{2} d x-\lambda \int_{\mathbb{R}^{N}} F(x, u) d x \\
& =\frac{1}{2}\left\|u^{+}\right\|^{2}-\frac{1}{2}\left\|u^{-}\right\|^{2}-\frac{\lambda-1}{2} \int_{\mathbb{R}^{N}} V_{-}(x) u^{2} d x-\lambda \int_{\mathbb{R}^{N}} F(x, u) d x .
\end{aligned}
$$


Let $u_{*} \in X$ and $\left\{u_{n}\right\} \subset X$ be such that $\left\|\left|u_{n}-u_{*} \|\right| \rightarrow 0\right.$. It follows that $u_{n}^{+} \rightarrow u_{*}^{+}, u_{n}^{-} \rightarrow u_{*}^{-}$, and $u_{n} \rightarrow u_{*}$. In addition, up to a subsequence, we can assume that $u_{n} \rightarrow u_{*}$ a.e. in $\mathbb{R}^{N}$. Then, we have

$$
\begin{aligned}
& \left\|u_{n}^{+}\right\|^{2} \rightarrow\left\|u_{*}^{+}\right\|^{2}, \\
& \liminf _{n \rightarrow \infty}\left\|u_{n}^{-}\right\|^{2} \geq\left\|u_{*}^{-}\right\|^{2}, \\
& \liminf _{n \rightarrow \infty} \int_{\mathbb{R}^{N}} V_{-}(x) u_{n}^{2} d x \geq \int_{\mathbb{R}^{N}} V_{-}(x) u_{*}^{2} d x \quad \text { (by the Fatou lemma). }
\end{aligned}
$$

By the definitions of $F$ and $\widetilde{F}$, it is easy to verify that, for all $(x, t) \in\left(\mathbb{R}^{N} \times(\mathbb{R} \backslash\{0\})\right.$,

$$
\frac{\partial}{\partial t}\left(\frac{F(x, t)}{t^{2}}\right)=\frac{2 \widetilde{F}(x, t)}{t^{3}}
$$

Together with $f(x, t)=o(t)$ as $|t| \rightarrow 0$ and $\left(\mathbf{f}_{3}\right)$, this implies that $F(x, t) \geq 0$ for all $x$ and $t$. By the Fatou lemma,

$$
\liminf _{n \rightarrow \infty} \int_{\mathbb{R}^{N}} F\left(x, u_{n}\right) d x \geq \int_{\mathbb{R}^{N}} F\left(x, u_{*}\right) d x .
$$

Then, by (2.9), we obtain

$$
\limsup _{n \rightarrow \infty} \Phi_{\lambda}\left(u_{n}\right) \leq \Phi_{\lambda}\left(u_{*}\right)
$$

This implies that $\Phi_{\lambda}$ is $\tau$-sequentially upper semi-continuous.

If $u_{n} \rightarrow u_{*}$ in $X$, then, for any fixed $\varphi \in X$, as $n \rightarrow \infty$,

$$
\begin{aligned}
\left\langle\Phi_{\lambda}^{\prime}\left(u_{n}\right), \varphi\right\rangle & =\int_{\mathbb{R}^{N}}\left(\nabla u_{n} \nabla \varphi+V_{\lambda} u_{n} \varphi\right) d x-\lambda \int_{\mathbb{R}^{N}} f\left(x, u_{n}\right) \varphi d x \\
& \rightarrow \int_{\mathbb{R}^{N}}\left(\nabla u_{*} \nabla \varphi+V_{\lambda} u_{*} \varphi\right) d x-\lambda \int_{\mathbb{R}^{N}} f\left(x, u_{*}\right) \varphi d x \\
& =\left\langle\Phi_{\lambda}^{\prime}\left(u_{*}\right), \varphi\right\rangle .
\end{aligned}
$$

This implies that $\Phi_{\lambda}^{\prime}$ is weakly sequentially continuous. Moreover, it is easy to see that $\Phi_{\lambda}$ maps bounded sets to bounded sets. Therefore, $\Phi_{\lambda}$ satisfies assumption $(c)$ in Proposition 2.2.

Finally, we shall verify assumption $(d)$ in Proposition 2.2 for $\Phi_{\lambda}$.

From (2.9), we have

$$
\Phi_{\lambda}(u)=\Phi(u)-\frac{\lambda-1}{2} \int_{\mathbb{R}^{N}} V_{-}(x) u^{2} d x-(\lambda-1) \int_{\mathbb{R}^{N}} F(x, u) d x, \quad \forall u \in X .
$$

From [15] (see also [6, Lemma 3.1 and Lemma 3.2]), we know that, under assumptions (v) and $\left(\mathbf{f}_{\mathbf{1}}\right)-\left(\mathbf{f}_{\mathbf{3}}\right)$, there exist $u_{0} \in X^{+} \backslash\{0\}$ with $\left\|u_{0}\right\|=1, \beta>0$, and $R>r>0$ such that

$$
\inf _{N} \Phi \geq \beta \quad \text { and } \quad \sup _{\partial M} \Phi \leq 0
$$

Let $K_{*}>1$ be chosen such that

$$
\left(K_{*}-1\right) \sup _{u \in N}\left(\frac{1}{2} \int_{\mathbb{R}^{N}} V_{-}(x) u^{2} d x+\int_{\mathbb{R}^{N}} F(x, u) d x\right)<\beta / 2 .
$$

Then, by (2.10) and $\inf _{N} \Phi \geq \beta$, we have that

$$
\inf _{N} \Phi_{\lambda} \geq \beta / 2, \quad \forall \lambda \in\left[1, K_{*}\right]
$$


Moreover, by (2.10) and $\sup _{\partial M} \Phi \leq 0$, we have that

$$
\sup _{\partial M} \Phi_{\lambda} \leq 0, \quad \forall \lambda \geq 1
$$

Together with (2.12), this implies that $\Phi_{\lambda}$ satisfies assumption $(d)$ in Proposition 2.2 if $\lambda \in$ $\left[1, K_{*}\right]$. Therefore, for $\lambda \in\left[1, K_{*}\right], \Phi_{\lambda}$ satisfies assumptions $(a)-(d)$ in Proposition 2.2. Then, the results of this lemma follow immediately from Proposition 2.2 .

Lemma 2.4. Suppose that $(\mathbf{v})$ and $\left(\mathbf{f}_{\mathbf{1}}\right)-\left(\mathbf{f}_{\mathbf{3}}\right)$ are satisfied. Let $\lambda \in\left[1, K_{*}\right]$ be fixed, where $K_{*}$ is the constant in Lemma 2.3. If $\left\{v_{n}\right\}$ is a bounded $(C)_{c}$ sequence for $\Phi_{\lambda}$ with $c \neq 0$, then for every $n \in \mathbb{N}$, there exists $a_{n} \in \mathbb{Z}^{N}$ such that, up to a subsequence, $u_{n}:=v_{n}\left(\cdot+a_{n}\right)$ satisfies

$$
u_{n} \rightarrow u_{\lambda} \neq 0, \quad \Phi_{\lambda}\left(u_{\lambda}\right) \leq c \quad \text { and } \quad \Phi_{\lambda}^{\prime}\left(u_{\lambda}\right)=0 .
$$

Proof. The proof of this lemma is inspired by the proof of Lemma 3.7 in [27]. Because $\left\{v_{n}\right\}$ is a bounded sequence in $X$, up to a subsequence, either

(a) $\lim _{n \rightarrow \infty} \sup _{y \in \mathbb{R}^{N}} \int_{B_{1}(y)}\left|v_{n}\right|^{2} d x=0$, or

(b) there exist $\rho>0$ and $a_{n} \in \mathbb{Z}^{N}$ such that $\int_{B_{1}\left(a_{n}\right)}\left|v_{n}\right|^{2} d x \geq \rho$.

If (a) occurs, using the Lions lemma (see, for example, [30, Lemma 1.21]), a similar argument as for the proof of [27, Lemma 3.6] shows that

$$
\lim _{n \rightarrow \infty} \int_{\mathbb{R}^{N}} F\left(x, v_{n}\right) d x=0 \quad \text { and } \quad \lim _{n \rightarrow \infty} \int_{\mathbb{R}^{N}} f\left(x, v_{n}\right) v_{n}^{ \pm} d x=0 .
$$

It follows that

$$
\lim _{n \rightarrow \infty} \int_{\mathbb{R}^{N}}\left(2 F\left(x, v_{n}\right)-f\left(x, v_{n}\right) v_{n}\right) d x=0 .
$$

On the other hand, as $\left\{v_{n}\right\}$ is a $(C)_{c}$-sequence of $\Phi_{\lambda}$, we have $\left\langle\Phi_{\lambda}^{\prime}\left(v_{n}\right), v_{n}\right\rangle \rightarrow 0$ and $\Phi_{\lambda}\left(v_{n}\right) \rightarrow$ $c \neq 0$. It follows that

$$
\begin{aligned}
& \int_{\mathbb{R}^{N}}\left(2 F\left(x, v_{n}\right)-f\left(x, v_{n}\right) v_{n}\right) d x \\
= & 2 \Phi_{\lambda}\left(v_{n}\right)-\left\langle\Phi_{\lambda}^{\prime}\left(v_{n}\right), v_{n}\right\rangle \rightarrow 2 c \neq 0, \quad n \rightarrow \infty .
\end{aligned}
$$

This contradicts (2.15). Therefore, case $(a)$ cannot occur.

If case $(b)$ occurs, let $u_{n}=v_{n}\left(\cdot+a_{n}\right)$. For every $n$,

$$
\int_{B_{1}(0)}\left|u_{n}\right|^{2} d x \geq \rho
$$

Because $V$ and $F(x, t)$ are 1-periodic in every $x_{j},\left\{u_{n}\right\}$ is still bounded in $X$,

$$
\lim _{n \rightarrow \infty} \Phi_{\lambda}\left(u_{n}\right) \leq c \quad \text { and } \quad \Phi_{\lambda}^{\prime}\left(u_{n}\right) \rightarrow 0, \quad n \rightarrow \infty .
$$

Up to a subsequence, we assume that $u_{n} \rightarrow u_{\lambda}$ in $X$ as $n \rightarrow \infty$. Since $u_{n} \rightarrow u_{\lambda}$ in $L_{l o c}^{2}\left(\mathbb{R}^{N}\right)$, it follows from (2.17) that $u_{\lambda} \neq 0$. Recall that $\Phi_{\lambda}^{\prime}\left(u_{n}\right)$ is weakly sequentially continuous. Therefore, $\Phi_{\lambda}^{\prime}\left(u_{n}\right) \rightarrow \Phi_{\lambda}^{\prime}\left(u_{\lambda}\right)$ and, by (2.18),$\Phi_{\lambda}^{\prime}\left(u_{\lambda}\right) \stackrel{\lambda}{=} 0$. 
Lemma 2.5. There exist $K_{* *}>1$ and $\eta>0$ such that for any $\lambda \in\left[1, K_{* *}\right]$, if $u \neq 0$ satisfies $\Phi_{\lambda}^{\prime}(u)=0$, then $\|u\| \geq \eta$.

Proof. We adapt the arguments of Yang [32, p. 2626] and Liu [17, Lemma 2.2]. Let $q=$ $(2 N-2) /(N-2)$ if $N \geq 3$ and $q=4$ if $N=1,2$. Note that by $\left(\mathbf{f}_{\mathbf{1}}\right)$ and $\left(\mathbf{f}_{\mathbf{2}}\right)$, for any $\varepsilon>0$, there exists $C_{\varepsilon}>0$ such that

$$
|f(x, t)| \leq \varepsilon|t|+C_{\varepsilon}|t|^{q-1} .
$$

Let $u \neq 0$ be a critical point of $\Phi_{\lambda}$. Then, by (2.9) and $\left\langle\Phi_{\lambda}^{\prime}(u), u^{ \pm}\right\rangle=0$, we have that

$$
\begin{aligned}
\left\|u^{ \pm}\right\|^{2}= & \pm(\lambda-1) \int_{\mathbb{R}^{N}} V_{-}(x) u u^{ \pm} d x \pm \lambda \int_{\mathbb{R}^{N}} f(x, u) u^{ \pm} d x \\
\leq & (\lambda-1) \sup _{\mathbb{R}^{N}} V_{-} \int_{\mathbb{R}^{N}}|u| \cdot\left|u^{ \pm}\right| d x \\
& +\varepsilon \int_{\mathbb{R}^{N}}|u| \cdot\left|u^{ \pm}\right| d x+C_{\varepsilon} \int_{\mathbb{R}^{N}}|u|^{q-1}\left|u^{ \pm}\right| d x \\
\leq & C_{1}((\lambda-1)+\varepsilon)|| u\|\cdot\| u^{ \pm}||+C_{2}|| u\left\|^{p-1}|| u^{ \pm}\right\|,
\end{aligned}
$$

where $C_{1}$ and $C_{2}$ are positive constants related to the Sobolev inequalities, and $\sup _{\mathbb{R}^{N}} V_{-}$. From the above two inequalities, we obtain

$$
\|u\|^{2}=\left\|u^{+}\right\|^{2}+\left\|u^{-}\right\|^{2} \leq 2 C_{1}((\lambda-1)+\varepsilon)\|u\|^{2}+2 C_{2}\|u\|^{p} .
$$

Because $p>2$, this implies that $\|u\| \geq \eta$ for some $\eta>0$ if $\varepsilon>0$ and $K_{* *}-1>0$ are small enough and $\lambda \in\left[1, K_{* *}\right]$. The desired result follows.

Let $K=\min \left\{K_{*}, K_{* *}\right\}$, where $K_{*}$ and $K_{* *}$ are the constants that appeared in Lemma2.3 and Lemma 2.5, respectively. Combining Lemmas 2.3 -2.5, we obtain the following lemma:

Lemma 2.6. Suppose $(\mathbf{v})$ and $\left(\mathbf{f}_{\mathbf{1}}\right)-\left(\mathbf{f}_{\mathbf{3}}\right)$ are satisfied. Then, there exist $\eta>0,\left\{\lambda_{n}\right\} \subset[1, K]$, and $\left\{u_{n}\right\} \subset X$ such that $\lambda_{n} \rightarrow 1$,

$$
\sup _{n} \Phi_{\lambda_{n}}\left(u_{n}\right)<+\infty, \quad\left\|u_{n}\right\| \geq \eta, \quad \text { and } \quad \Phi_{\lambda_{n}}^{\prime}\left(u_{n}\right)=0 .
$$

\section{Boundedness of approximate solutions and proofs of the main results}

In this section, we show that the sequence of approximate solutions $\left\{u_{n}\right\}$ obtained in Lemma 2.6 is bounded in $X$. We then give the proofs of Theorem 1.1 and Corollary 1.2 .

Lemma 3.1. Suppose $(\mathbf{v}),\left(\mathbf{v}^{\prime}\right)$, and $\left(\mathbf{f}_{\mathbf{1}}\right)-\left(\mathbf{f}_{\mathbf{3}}\right)$ are satisfied. Let $\left\{u_{n}\right\}$ be the sequence obtained in Lemma 2.6. Then, $\left\{u_{n}\right\} \subset L^{\infty}\left(\mathbb{R}^{N}\right)$ and

$$
\sup _{n}\left\|u_{n}\right\|_{L^{\infty}\left(\mathbb{R}^{N}\right)}<+\infty .
$$

Proof. From $\Phi_{\lambda_{n}}^{\prime}\left(u_{n}\right)=0$, we deduce that $u_{n}$ is a weak solution of (2.6) with $\lambda=\lambda_{n}$, i.e.,

$$
-\Delta u_{n}+V_{\lambda_{n}}(x) u_{n}=\lambda_{n} f\left(x, u_{n}\right) \quad \text { in } \quad \mathbb{R}^{N} .
$$

Because $f \in C\left(\mathbb{R}^{N} \times \mathbb{R}\right)$ and it is asymptotically linear, we can use the bootstrap argument of elliptic equations to deduce that $u_{n} \in L^{\infty}\left(\mathbb{R}^{N}\right)$ and is Hölder continuous. For every $a \in \mathbb{Z}^{N}$, 
$u_{n}(\cdot+a)$ is still a solution of (3.2), and so, without loss of generality, we assume that for every $n \in \mathbb{N}$, there exists $x_{n} \in \mathbb{R}^{N}$ with $\left|x_{n}\right| \leq 1$ such that

$$
\left|u_{n}\left(x_{n}\right)\right|=\max _{x \in \mathbb{R}^{N}}\left|u_{n}(x)\right|=\|\left. u_{n}\right|_{L^{\infty}\left(\mathbb{R}^{N}\right)} .
$$

If (3.1) were not true, then $\left\|u_{n}\right\|_{L^{\infty}\left(\mathbb{R}^{N}\right)} \rightarrow+\infty$. Denote $v_{n}=u_{n} /\left\|u_{n}\right\|_{L^{\infty}\left(\mathbb{R}^{N}\right)}$. Then, for every $x \in \mathbb{R}^{N},\left|v_{n}(x)\right| \leq 1$, and for every $n,\left|v_{n}\left(x_{n}\right)\right|=1$. Moreover, $v_{n}$ satisfies

$$
-\Delta v_{n}+V_{\lambda_{n}}(x) v_{n}=\lambda_{n} \frac{f\left(x, u_{n}\right)}{u_{n}} v_{n} \quad \text { in } \quad \mathbb{R}^{N}
$$

$\operatorname{Assup}_{x \in \mathbb{R}^{N}, n \in \mathbb{N}}\left|V_{\lambda_{n}}(x)\right|<+\infty$, and $\sup _{x \in \mathbb{R}^{N}, n \in \mathbb{N}} \lambda_{n}\left|f\left(x, u_{n}(x)\right)\right| /\left|u_{n}(x)\right|<+\infty$ (by $\left(\mathbf{f}_{\mathbf{1}}\right)$ and $\left(\mathbf{f}_{\mathbf{3}}\right)$ ), we use the $L^{p}$-estimate of elliptic equations (see, for example, [8]) to deduce that for any $p>2$ and $R>0$, there exists $C_{R}>0$ such that

$$
\left\|v_{n}\right\|_{W^{2, p}\left(B_{R}(0)\right)} \leq C_{R}\left\|v_{n}\right\|_{L^{p}\left(B_{R+1}(0)\right)}
$$

For any $x \in \mathbb{R}^{N},\left|v_{n}(x)\right| \leq 1$, which implies that $\|\left. v_{n}\right|_{L^{p}\left(B_{R+1}(0)\right)} \leq\left|B_{R+1}(0)\right|^{1 / p}$, where $|A|$ denotes the Lebesgue measure of a set $A \subset \mathbb{R}^{N}$. Therefore, for any $R>0$, there exists $D_{R}>0$ such that

$$
\left\|v_{n}\right\|_{W^{2, p}\left(B_{R}(0)\right)} \leq D_{R}
$$

Taking $p>N$ in (3.6), from the Sobolev embedding theorem (see, for example, [2, Chapter 4]), we deduce that there exists $C_{R}^{\prime}>0$ such that

$$
\left\|v_{n}\right\|_{C^{1, \alpha}\left(\overline{B_{R}(0)}\right)} \leq C_{R}^{\prime}\left\|v_{n}\right\|_{W^{2, p}\left(B_{R}(0)\right)} \leq C_{R}^{\prime} D_{R}
$$

where $\alpha=1-N / p$. For every $R>0$, the embedding $C^{1, \alpha}\left(\overline{B_{R}(0)}\right) \hookrightarrow C^{1}\left(\overline{B_{R}(0)}\right)$ is compact, and so we can use the diagonal process to deduce that there exist a subsequence $\left\{v_{n_{m}}\right\}$ of $\left\{v_{n}\right\}$ and $v \in C^{1}\left(\mathbb{R}^{N}\right)$, such that, for every $k \in \mathbb{N}$,

$$
v_{n_{m}} \rightarrow v \quad \text { in } \quad C^{1}\left(\overline{B_{k}(0)}\right), \quad \text { as } \quad m \rightarrow \infty .
$$

It follows that

$$
v_{n_{m}} \rightarrow v \text {, a.e. in } \mathbb{R}^{N}, \quad \text { as } \quad m \rightarrow \infty .
$$

Because $\left|v_{n_{m}}(x)\right| \leq 1, \forall x \in \mathbb{R}^{N}$, (3.9) implies that $|v(x)| \leq 1, \forall x \in \mathbb{R}^{N}$. In addition, from $\left|v_{n_{m}}\left(x_{n_{m}}\right)\right|=1$ and $\left|x_{n_{m}}\right| \leq 1, m=1,2, \cdots$, we deduce that there exists $x_{0} \in \mathbb{R}^{N}$ with $\left|x_{0}\right| \leq 1$ such that, up to a subsequence, $x_{n_{m}} \rightarrow x_{0}$ as $m \rightarrow \infty$ and $\left|v\left(x_{0}\right)\right|=1$.

As the sequence $\left\{h_{n}\right\}$ defined by

$$
h_{n}(x)= \begin{cases}f\left(x, u_{n}(x)\right) / u_{n}(x), & u_{n}(x) \neq 0 \\ 0, & u_{n}(x)=0\end{cases}
$$

is bounded in $L^{\infty}\left(\mathbb{R}^{N}\right)$, and $L^{\infty}\left(\mathbb{R}^{N}\right)$ is the dual space of $L^{1}\left(\mathbb{R}^{N}\right)$, the Banach-Alaoglu theorem (see, for example, [20, Theorem 3.15]) implies that, up to a subsequence, $h_{n}$ converges in the weak* topology to some function $h \in L^{\infty}\left(\mathbb{R}^{N}\right)$, i.e., for any $g \in L^{1}\left(\mathbb{R}^{N}\right)$,

$$
\int_{\mathbb{R}^{N}} h_{n}(x) g(x) d x \rightarrow \int_{\mathbb{R}^{N}} h(x) g(x) d x, \quad n \rightarrow \infty .
$$


Then, by $v_{n_{m}} \rightarrow v$ in $C_{l o c}^{1}\left(\mathbb{R}^{N}\right)$ (see (3.8)), we have that, for any $\varphi \in C_{0}^{\infty}\left(\mathbb{R}^{N}\right)$, as $m \rightarrow \infty$,

$$
\begin{aligned}
& \left|\int_{\mathbb{R}^{N}} h_{n_{m}} v_{n_{m}} \varphi d x-\int_{\mathbb{R}^{N}} h v \varphi d x\right| \\
\leq & \int_{\text {supp } \varphi}\left|h_{n_{m}}\right| \cdot\left|v_{n_{m}}-v\right| \cdot|\varphi| d x+\left|\int_{\mathbb{R}^{N}} h_{n_{m}} v \varphi d x-\int_{\mathbb{R}^{N}} h v \varphi d x\right| \rightarrow 0,
\end{aligned}
$$

where $\operatorname{supp} \varphi$ denotes the support of $\varphi$. For any $\varphi \in C_{0}^{\infty}\left(\mathbb{R}^{N}\right)$, we have

$$
\int_{\mathbb{R}^{N}} \nabla v_{n_{m}} \nabla \varphi d x+\int_{\mathbb{R}^{N}} V_{\lambda_{n_{m}}}(x) v_{n_{m}} \varphi d x=\lambda_{n_{m}} \int_{\mathbb{R}^{N}} h_{n_{m}}(x) v_{n_{m}} \varphi d x .
$$

As $m \rightarrow \infty$, we have

$$
\begin{aligned}
& \lambda_{n_{m}} \rightarrow 1 \\
& \int_{\mathbb{R}^{N}} \nabla v_{n_{m}} \nabla \varphi d x \rightarrow \int_{\mathbb{R}^{N}} \nabla v \nabla \varphi d x, \\
& \int_{\mathbb{R}^{N}} V_{\lambda_{n_{m}}}(x) v_{n_{m}} \varphi d x \rightarrow \int_{\mathbb{R}^{N}} V(x) v \varphi d x,
\end{aligned}
$$

and therefore,

$$
\int_{\mathbb{R}^{N}} \nabla v \nabla \varphi d x+\int_{\mathbb{R}^{N}} V(x) v \varphi d x=\int_{\mathbb{R}^{N}} h(x) v \varphi d x, \quad \forall \varphi \in C_{0}^{\infty}\left(\mathbb{R}^{N}\right) .
$$

It follows that $v$ solves the linear problem

$$
-\Delta v+V(x) v=h(x) v \quad \text { in } \quad \mathbb{R}^{N} .
$$

Because $v \in C^{1}\left(\mathbb{R}^{N}\right)$ and $\left|v\left(x_{0}\right)\right|=1$, we can deduce that $v \neq 0$. Moreover, as $h \in L^{\infty}\left(\mathbb{R}^{N}\right)$, by the regularity theorem of elliptic equations (see, for example, [8]), we have that $v \in W_{l o c}^{2,2}\left(\mathbb{R}^{N}\right)$. Then, by the strong unique continuation property (as in [12, Theorem 6.3]), $v(x) \neq 0$ a.e. in $\mathbb{R}^{N}$, which implies $\left|u_{n_{m}}(x)\right| \rightarrow+\infty$, a.e. in $\mathbb{R}^{N}$. Hence, from $\left(\mathbf{f}_{\mathbf{2}}\right)$, we have that $h_{n_{m}}(x) \rightarrow V_{\infty}(x)$ a.e. in $\mathbb{R}^{N}$.

We now prove that $h=V_{\infty}$. It suffices to prove that, for any $\varphi \in C_{0}^{\infty}\left(\mathbb{R}^{N}\right)$,

$$
\int_{\mathbb{R}^{N}} h \varphi d x=\int_{\mathbb{R}^{N}} V_{\infty} \varphi d x
$$

By the Egoroff theorem (see, for example, [21]) and $h_{n_{m}}(x) \rightarrow V_{\infty}(x)$ a.e. in $\mathbb{R}^{N}$, we deduce that, for any $\varepsilon>0$, there exists a measurable set $E_{\varepsilon} \subset \operatorname{supp} \varphi$ such that $\left|\operatorname{supp} \varphi \backslash E_{\varepsilon}\right|<\varepsilon$ and $h_{n_{m}}$ converges uniformly to $V_{\infty}$ on $E_{\varepsilon}$. This implies that

$$
\lim _{m \rightarrow \infty} \int_{E_{\varepsilon}}\left|h_{n_{m}}-V_{\infty}\right| \cdot|\varphi| d x=0
$$

and

$$
\begin{aligned}
& \sup _{m} \int_{\operatorname{supp} \varphi \backslash E_{\varepsilon}}\left|h_{n_{m}}-V_{\infty}\right| \cdot|\varphi| d x \\
\leq & \left.\sup _{m}\left\|h_{n_{m}}\right\|_{L^{\infty}\left(\mathbb{R}^{N}\right)}+|| V_{\infty} \|_{L^{\infty}\left(\mathbb{R}^{N}\right)}\right) \int_{\text {supp } \varphi \backslash E_{\varepsilon}}|\varphi| d x \leq C \varepsilon,
\end{aligned}
$$

where $C>0$ is a constant independent of $m$. Therefore,

$$
\limsup _{n \rightarrow \infty} \int_{\mathbb{R}^{N}}\left|h_{n_{m}}-V_{\infty}\right| \cdot|\varphi| d x
$$




$$
\begin{aligned}
\leq & \limsup _{n \rightarrow \infty} \int_{E_{\varepsilon}}\left|h_{n_{m}}-V_{\infty}\right| \cdot|\varphi| d x \\
& +\limsup _{n \rightarrow \infty} \int_{\text {supp } \varphi \backslash E_{\varepsilon}}\left|h_{n_{m}}-V_{\infty}\right| \cdot|\varphi| d x \leq C \varepsilon .
\end{aligned}
$$

Letting $\varepsilon \rightarrow 0$, we get (3.13). Therefore, $v \in L^{\infty}\left(\mathbb{R}^{N}\right) \cap C^{1}\left(\mathbb{R}^{N}\right)$ is a nonzero solution of the linear problem

$$
-\Delta u+\left(V(x)-V_{\infty}(x)\right) u=0 \quad \text { in } \quad \mathbb{R}^{N} .
$$

For $1 \leq p \leq \infty$, let $T_{p}$ be the operator

$$
\begin{aligned}
& T_{p}: L^{p}\left(\mathbb{R}^{N}\right) \rightarrow L^{p}\left(\mathbb{R}^{N}\right), \quad u \mapsto-\Delta u+\left(V-V_{\infty}\right) u, \\
& \text { with domain } D\left(T_{p}\right):=\left\{u \in L^{p}\left(\mathbb{R}^{N}\right) \mid T_{p} u \in L^{p}\left(\mathbb{R}^{N}\right)\right\} .
\end{aligned}
$$

Because $V-V_{\infty} \in L^{\infty}\left(\mathbb{R}^{N}\right)$, it was proved in [10] that $\sigma\left(T_{p}\right)$, the spectrum of $T_{p}$, is independent of $p \in[1,+\infty]$. In particular, we have $\sigma\left(T_{2}\right)=\sigma\left(T_{\infty}\right)$. Assumption $(\mathbf{v})$ implies that $0 \notin \sigma\left(T_{2}\right)$. Consequently, $0 \notin \sigma\left(T_{\infty}\right)$. However, as $v \in L^{\infty}\left(\mathbb{R}^{N}\right)$ is a nonzero solution of (3.14), we deduce that $0 \in \sigma\left(T_{\infty}\right)$. This induces a contradiction. Therefore, $\sup _{n}\left\|u_{n}\right\|_{L^{\infty}\left(\mathbb{R}^{N}\right)}<+\infty$, which completes the proof.

Remark 3.2. From Theorem 1.2 in [24] or Theorem C.4.2 in [25], we can also deduce that if (3.14) has a nonzero solution $v \in L^{\infty}\left(\mathbb{R}^{N}\right) \cap C^{1}\left(\mathbb{R}^{N}\right)$, then $0 \in \sigma\left(T_{2}\right)$.

Lemma 3.3. Suppose that $(\mathbf{v}),\left(\mathbf{v}^{\prime}\right),\left(\mathbf{f}_{\mathbf{1}}\right)-\left(\mathbf{f}_{\mathbf{3}}\right)$, and $\left(\mathbf{f}_{\mathbf{4}}^{\prime}\right)$ are satisfied. Let $\left\{u_{n}\right\}$ be the sequence obtained in Lemma 2.6 Then

$$
0<\inf _{n}\left\|u_{n}\right\| \leq \sup _{n}\left\|u_{n}\right\|<+\infty .
$$

Proof. As $\Phi_{\lambda_{n}}^{\prime}\left(u_{n}\right)=0$ and $u_{n} \neq 0$, Lemma 2.5 implies that $\inf _{n}\left\|u_{n}\right\|>0$.

To prove $\sup _{n}|| u_{n} \|<+\infty$, we apply an indirect argument, and assume by contradiction that $\left\|u_{n}\right\| \rightarrow+\infty$. have

Since $\Phi_{\lambda_{n}}^{\prime}\left(u_{n}\right)=0$, by (2.19) and $\left|f\left(x, u_{n}\right)\right| \leq C\left|u_{n}\right|$ for some constant $C>0$ (see $\left(\mathbf{f}_{2}\right)$ ), we

$$
\begin{aligned}
0 & = \pm\left\|u_{n}^{ \pm}\right\|^{2}-\left(\lambda_{n}-1\right) \int_{\mathbb{R}^{N}} V_{-}(x) u_{n} u_{n}^{ \pm} d x-\lambda_{n} \int_{\mathbb{R}^{N}} f\left(x, u_{n}\right) u_{n}^{ \pm} d x \\
& = \pm\left\|u_{n}^{ \pm}\right\|^{2}-\int_{\mathbb{R}^{N}} f\left(x, u_{n}\right) u_{n}^{ \pm} d x+\left(\lambda_{n}-1\right) O\left(\left\|u_{n}\right\|^{2}\right) .
\end{aligned}
$$

It follows that

$$
\begin{aligned}
& \left\|u_{n}\right\|^{2}-\int_{\mathbb{R}^{N}} f\left(x, u_{n}\right)\left(u_{n}^{+}-u_{n}^{-}\right) d x \\
= & \left\|u_{n}^{+}\right\|^{2}+\left\|u_{n}^{-}\right\|^{2}-\int_{\mathbb{R}^{N}} f\left(x, u_{n}\right)\left(u_{n}^{+}-u_{n}^{-}\right) d x=\left(\lambda_{n}-1\right) O\left(\left\|u_{n}\right\|^{2}\right) .
\end{aligned}
$$

Set $w_{n}=u_{n} /\left\|u_{n}\right\|$. Then by (3.16),

$$
\left\|u_{n}\right\|^{2}\left(1-\int_{\mathbb{R}^{N}} \frac{f\left(x, u_{n}\right)}{u_{n}}\left(w_{n}^{+}-w_{n}^{-}\right) w_{n} d x\right)=\left(\lambda_{n}-1\right) O\left(\left\|u_{n}\right\|^{2}\right) .
$$

And by $\lambda_{n} \rightarrow 1$ as $n \rightarrow \infty$, we have that

$$
\int_{\mathbb{R}^{N}} \frac{f\left(x, u_{n}\right)}{u_{n}}\left(w_{n}^{+}-w_{n}^{-}\right) w_{n} d x \rightarrow 1, \quad n \rightarrow \infty .
$$


From Lemma 2.6,

$$
C_{0}:=\sup _{n} \Phi_{\lambda_{n}}\left(u_{n}\right)<+\infty .
$$

Then, by $\Phi_{\lambda_{n}}^{\prime}\left(u_{n}\right)=0$ and $\int_{\mathbb{R}^{N}} \widetilde{F}\left(x, u_{n}\right) d x=O\left(\left\|u_{n}\right\|^{2}\right)$, we obtain

$$
\begin{aligned}
C_{0} & \geq 2 \Phi_{\lambda_{n}}\left(u_{n}\right)-\left\langle\Phi_{\lambda_{n}}^{\prime}\left(u_{n}\right), u_{n}\right\rangle \\
& =2 \lambda_{n} \int_{\mathbb{R}^{N}} \widetilde{F}\left(x, u_{n}\right) d x \\
& =\left(\lambda_{n}-1\right) O\left(\left\|u_{n}\right\|^{2}\right)+2 \int_{\mathbb{R}^{N}} \widetilde{F}\left(x, u_{n}\right) d x
\end{aligned}
$$

Together with $\left(\mathbf{f}_{\mathbf{3}}\right)$, this implies

$$
\left(\lambda_{n}-1\right) O\left(\left\|u_{n}\right\|^{2}\right)+C_{0} \geq 2 \int_{\mathbb{R}^{N}} \widetilde{F}\left(x, u_{n}\right) d x \geq 2 \int_{\left\{x|b \geq| u_{n}(x) \mid \geq \kappa\right\}} \widetilde{F}\left(x, u_{n}\right) d x
$$

where

$$
b:=\sup _{n}\left\|u_{n}\right\|_{L^{\infty}\left(\mathbb{R}^{N}\right)} \cdot
$$

From Lemma 3.1 we have $b<+\infty$. As the continuous function $\widetilde{F}$ is 1-periodic in every $x_{j}$ variable, we deduce from (1.4) that there exists a constant $C^{\prime}>0$ such that

$$
\widetilde{F}(x, t) \geq C^{\prime} t^{2}, \quad \text { for all } \quad \kappa \leq|t| \leq b \quad \text { and } \quad x \in \mathbb{R}^{N} .
$$

Combining (3.18) and (3.19) leads to

$$
\left(\lambda_{n}-1\right) O\left(\left\|u_{n}\right\|^{2}\right)+C_{0} \geq 2 C^{\prime} \int_{\left\{x|b \geq| u_{n}(x) \mid \geq \kappa\right\}} u_{n}^{2} d x .
$$

Dividing both sides of this inequality by $\left\|u_{n}\right\|^{2}$ and sending $n \rightarrow \infty$, we obtain

$$
\lim _{n \rightarrow \infty} \int_{\left\{x|b \geq| u_{n}(x) \mid \geq \kappa\right\}} w_{n}^{2} d x=0 .
$$

From (1.3), (2.2), and (2.3), we have that

$$
\begin{aligned}
& \int_{\left\{x|| u_{n}(x) \mid<\kappa\right\}}\left|\frac{f\left(x, u_{n}\right)}{u_{n}}\left(w_{n}^{+}-w_{n}^{-}\right) w_{n}\right| d x \\
\leq & v \int_{\left\{x|| u_{n}(x) \mid<\kappa\right\}}\left|\left(w_{n}^{+}-w_{n}^{-}\right) w_{n}\right| d x \\
\leq & v \int_{\mathbb{R}^{N}}\left|\left(w_{n}^{+}-w_{n}^{-}\right) w_{n}\right| d x \\
\leq & v\left\|w_{n}\right\|_{L^{2}}^{2} \leq \frac{v}{\mu_{0}}\left\|w_{n}\right\|^{2}=\frac{v}{\mu_{0}}<1 .
\end{aligned}
$$

Because $\left|f\left(x, u_{n}\right)\right| \leq C\left|u_{n}\right|$ for some constant $C>0$ (see $\left(\mathbf{f}_{\mathbf{2}}\right)$ ), (3.20) gives

$$
\begin{aligned}
& \int_{\left\{x|b \geq| u_{n}(x) \mid \geq \kappa\right\}}\left|\frac{f\left(x, u_{n}\right)}{u_{n}}\left(w_{n}^{+}-w_{n}^{-}\right) w_{n}\right| d x \\
\leq & C \int_{\left\{x|b \geq| u_{n}(x) \mid \geq \kappa\right\}}\left|\left(w_{n}^{+}-w_{n}^{-}\right) w_{n}\right| d x \\
\leq & C|| w_{n}^{+}-w_{n}^{-}||_{L^{2}}\left(\int_{\left\{x|b \geq| u_{n}(x) \mid \geq \kappa\right\}} w_{n}^{2} d x\right)^{1 / 2}
\end{aligned}
$$




$$
\leq C|| w_{n} \|_{L^{2}}\left(\int_{\left\{x|b \geq| u_{n}(x) \mid \geq \kappa\right\}} w_{n}^{2} d x\right)^{1 / 2} \rightarrow 0, \quad n \rightarrow \infty .
$$

Combining (3.21) and (3.22) yields

$$
\begin{aligned}
& \limsup _{n \rightarrow \infty} \int_{\mathbb{R}^{N}}\left|\frac{f\left(x, u_{n}\right)}{u_{n}}\left(w_{n}^{+}-w_{n}^{-}\right) w_{n}\right| d x \\
\leq & \limsup _{n \rightarrow \infty} \int_{\left\{x|| u_{n}(x) \mid<\kappa\right\}}\left|\frac{f\left(x, u_{n}\right)}{u_{n}}\left(w_{n}^{+}-w_{n}^{-}\right) w_{n}\right| d x \\
& +\limsup _{n \rightarrow \infty} \int_{\left\{x|b \geq| u_{n}(x) \mid \geq \kappa\right\}}\left|\frac{f\left(x, u_{n}\right)}{u_{n}}\left(w_{n}^{+}-w_{n}^{-}\right) w_{n}\right| d x<1 .
\end{aligned}
$$

This contradicts (3.17). Therefore, $\left\{u_{n}\right\}$ is bounded in $X$.

Proof of Theorem 1.1, Let $\left\{u_{n}\right\}$ be the sequence obtained in Lemma 2.6. From Lemma 3.3 , $\left\{u_{n}\right\}$ is bounded in $X$. Therefore, up to a subsequence, either

(a) $\lim _{n \rightarrow \infty} \sup _{y \in \mathbb{R}^{N}} \int_{B_{1}(y)}\left|u_{n}\right|^{2} d x=0$, or

(b) there exist $\rho>0$ and $y_{n} \in \mathbb{Z}^{N}$ such that $\int_{B_{1}\left(y_{n}\right)}\left|u_{n}\right|^{2} d x \geq \rho$.

According to (2.14), if case $(a)$ occurs,

$$
\lim _{n \rightarrow \infty} \int_{\mathbb{R}^{N}} f\left(x, u_{n}\right) u_{n}^{ \pm} d x=0 .
$$

Then, by (2.19) and $\lambda_{n} \rightarrow 1$, we have

$$
\begin{aligned}
\left\|u_{n}^{ \pm}\right\|^{2} & = \pm\left(\lambda_{n}-1\right) \int_{\mathbb{R}^{N}} V_{-}(x) u_{n} u_{n}^{ \pm} d x \pm \lambda_{n} \int_{\mathbb{R}^{N}} f\left(x, u_{n}\right) u_{n}^{ \pm} d x \\
& \leq C\left(\lambda_{n}-1\right)\left\|u_{n}\right\|_{L^{2}}^{2}+K\left|\int_{\mathbb{R}^{N}} f\left(x, u_{n}\right) u_{n}^{ \pm} d x\right| \rightarrow 0 .
\end{aligned}
$$

This contradicts $\inf _{n}\left\|u_{n}\right\|>0$ (see (3.15)). Therefore, case $(a)$ cannot occur. As case $(b)$ therefore occurs, the proof of Lemma 2.4 implies that there exists $y_{n} \in \mathbb{Z}^{N}$ such that $w_{n}=$ $u_{n}\left(\cdot+y_{n}\right)$ satisfies $w_{n} \rightarrow u_{0} \neq 0$. Because $\Phi_{\lambda_{n}}^{\prime}\left(u_{n}\right)=0$ (by Lemma 2.6), we have $\Phi_{\lambda_{n}}^{\prime}\left(w_{n}\right)=0$. From (2.10), we have that, for any $\varphi \in X$,

$$
\begin{aligned}
& \left\langle\Phi_{\lambda_{n}}^{\prime}\left(w_{n}\right), \varphi\right\rangle \\
= & \left\langle\Phi^{\prime}\left(w_{n}\right), \varphi\right\rangle-\left(\lambda_{n}-1\right) \int_{\mathbb{R}^{N}} V_{-}(x) w_{n} \varphi d x-\left(\lambda_{n}-1\right) \int_{\mathbb{R}^{N}} f\left(x, w_{n}\right) \varphi d x .
\end{aligned}
$$

Together with $\Phi_{\lambda_{n}}^{\prime}\left(w_{n}\right)=0$ and $\lambda_{n} \rightarrow 1$, this yields

$$
\left\langle\Phi^{\prime}\left(w_{n}\right), \varphi\right\rangle \rightarrow 0, \quad \forall \varphi \in X
$$

Finally, by $w_{n} \rightarrow u_{0} \neq 0$ and the weakly sequential continuity of $\Phi^{\prime}$, we have that $\Phi^{\prime}\left(u_{0}\right)=0$. Therefore, $u_{0}$ is a nontrivial solution of Eq.(1.1). This completes the proof.

Proof of Corollary 1.2. Assumption $\left(\mathbf{f}_{\mathbf{5}}^{\prime}\right)$ and the assumption that $f(x, t) / t \rightarrow 0$ uniformly in $x \in \mathbb{R}^{N}$ as $t \rightarrow 0$ imply $\left(\mathbf{f}_{\mathbf{4}}^{\prime}\right)$. Thus, this corollary follows immediately from Theorem 1.1 . 


\section{References}

[1] N. Ackermann, A superposition principle and multibump solutions of periodic Schrödinger equations. J. Func. Anal. 234 (2006) 277-320.

[2] R. Adams, J. F. Fournier, Sobolev Spaces. Second edition. Pure and Applied Mathematics (Amsterdam), 140. Elsevier/Academic Press, Amsterdam, 2003.

[3] S. Alama, Y. Y. Li, On multibump bound states for certain semilinear elliptic equations. Indiana Univ. Math. J. 41 (1992) 983-1026.

[4] V. Coti Zelati, P. Rabinowitz, Homoclinic type solutions for a semilinear elliptic PDE on $\mathbb{R}^{n}$, Comm. Pure Appl. Math. 45 (1992) 1217-1269.

[5] S. Chen, Multi-bump solutions for a strongly indefinite semilinear Schrödinger equation without symmetry or convexity assumptions, Nonlinear Anal. 68 (2008) 3067-3102.

[6] Y. H. Ding, C. Lee, Multiple solutions of Schrödinger equations with indefinite linear part and super or asymptotically linear terms, J. Differential Equations, 222 (2006) 137-163.

[7] Y. H. Ding, Variational Methods for Strongly Indefinite Problems. Interdisciplinary Mathematical Sciences, 7. World Scientific Publishing Co. Pte. Ltd., Hackensack, NJ, 2007.

[8] D. Gilbarg, N. Trudinger, Elliptic Partial Differential Equations of Second Order. Reprint of the 1998 edition. Classics in Mathematics. Springer-Verlag, Berlin, 2001.

[9] H.-P. Heinz, T. Küpper, C. A. Stuart, Existence and bifurcation of solutions for nonlinear perturbations of the periodic Schrödinger equation, J. Differential Equations 100 (1992) 341-354.

[10] R. Hempel, J. Voigt, The spectrum of a Schrödinger operator in $L_{p}\left(\mathbb{R}^{v}\right)$ is $p$-independent, Comm. Math. Phys., 104 (1986) 243-250.

[11] L. Jeanjean, On the existence of bounded Palais-Smale sequences and application to a Landesman-Lazer type problem set on $\mathbb{R}^{N}$, Proc. Roy. Soc. Edinb. 129A (1999) 787-809.

[12] D. Jerison, C. Kenig, Unique continuation and absence of positive eigenvalues for Schrödinger operators, Ann. of Math. (2) 121 (1985) 463-494.

[13] W. Kryszewski, A. Szulkin, Generalized linking theorem with an application to a semilinear Schrödinger equation, Adv. Differ. Equ. 3 (1998) 441-472.

[14] W. Kryszewski, A. Szulkin, Infinite-dimensional homology and multibump solutions, J. Fixed Point Theory Appl. 5 (2009) 1-35.

[15] G. B. Li, A. Szulkin, An asymptotically periodic Schrödinger equation with indefinite linear part, Commun. Contemp. Math., 4 (2002) 763-776.

[16] Y.Q. Li, Z.Q. Wang, J. Zeng, Ground states of nonlinear Schrödinger equations with potentials, Ann. Inst. H. Poincaré Anal. Non Linéaire, 23 (2006) 829-837.

[17] S. Liu, On superlinear Schrödinger equations with periodic potential, Calc. Var. Partial Differential Equations 45 (2012) 1-9.

[18] A. Pankov, K. Pflüger, On a semilinear Schrödinger equation with periodic potential, Nonlinear Anal. 33 (1998) 593-609. 
[19] A. Pankov, Periodic nonlinear Schrödinger equation with application to photonic crystals, Milan J. Math. 73 (2005) 259-287.

[20] W. Rudin, Functional Analysis. Second edition. International Series in Pure and Applied Mathematics. McGraw-Hill, Inc., New York, 1991.

[21] W. Rudin, Real and Complex Analysis. Second edition. McGraw-Hill Series in Higher Mathematics. McGraw-Hill Book Co., New York-Düsseldorf-Johannesburg, 1974.

[22] M. Schechter, Superlinear Schrödinger operators, J. Funct. Anal. 262 (2012) 2677-2694.

[23] M. Schechter, W. M. Zou, Weak linking theorems and Schrödinger equations with critical Sobolev exponent, ESAIM Control Optim. Calc. Var. 9 (2003) 601-619.

[24] B. Simon, Spectrum and continuum eigenfunctions of Schrödinger operators, J. Funct. Anal. 42 (1981), no. 3, 347-355.

[25] B. Simon, Schrödinger semigroups, Bull. Amer. Math. Soc. (N.S.) 7 (1982), no. 3, 447526.

[26] A. Szulkin, T. Weth, Ground state solutions for some indefinite variational problems, J. Funct. Anal. 257 (2009) 3802-3822.

[27] A. Szulkin, W. Zou, Homoclinic orbits for asymptotically linear Hamiltonian systems, J. Funct. Anal. 187 (2001) 25-41.

[28] C. Troestler, M. Willem, Nontrivial solution of a semilinear Schrödinger equation, Comm. Partial Differential Equations 21 (1996) 1431-1449.

[29] C. Troestler, Bifurcation into spectral gaps for a noncompact semilinear Schrödinger equation with nonconvex potential, preprint, see http://arxiv.org/pdf/1207.1052.pdf

[30] M. Willem, Minimax Theorems, Progress in Nonlinear Differential Equations and their Applications, Vol 24. Birkhäuser Boston, Inc., Boston, MA, 1996.

[31] M. Willem, W. Zou, On a Schrödinger equation with periodic potential and spectrum point zero, Indiana Univ. Math. J. 52 (2003) 109-132.

[32] M. Yang, Ground state solutions for a periodic Schrödinger equation with superlinear nonlinearities, Nonlinear Anal. 72 (2010) 2620-2627.

[33] M. Yang, W. Chen, Y. H. Ding, Solutions for periodic Schrödinger equation with spectrum zero and general superlinear nonlinearities, J. Math. Anal. Appl. 364 (2010) 404-413. 